\title{
Prevalence of iron deficiency anemia among pregnant women before iron supplementation in Kathmandu university Hospital/Dhulikhel Hospital
}

\author{
Binay Kumar Raut ${ }^{1}$, Mukesh Kumar Jha ${ }^{2}$, Abha Shrestha ${ }^{3}$, Ajya Sah $^{4}$, Amit Sapkota ${ }^{4}$, \\ Smarika Byanju ${ }^{4}$, Shyam Sundar Malla ${ }^{1}$ \\ ${ }^{1}$ Department of Biochemistry, Kathmandu University School of Medical Sciences, Dhulikhel, Kavre, Nepal \\ ${ }^{2}$ Department of Physiology, Kathmandu University School of Medical Sciences, Dhulikhel, Kavre, Nepal \\ ${ }^{3}$ Department of Obstetrics and Gynecology, Kathmandu University School of Medical Sciences, Dhulikhel, Kavre, Nepal \\ ${ }^{4}$ Faculty of Human Biology, Kathmandu University School of Medical Sciences, Dhulikhel, Kavre, Nepal
}

\section{Email address:}

binaymolbio@gmail.com (B. K. Raut),jhamukesh.jnp@gmail.com (M. K. Jha),phuche_001@yahoo.com (A. Shrestha), ajaywishes2@gmail.com (A. Sah), amitsapkota09@gmail.com (A. Sapkota), smarikasapkota03@gmail.com (S. Byanju), shyam_malla@hotmail.com (S. S. Malla)

\section{To cite this article:}

Binay Kumar Raut, Mukesh Kumar Jha, Abha Shrestha, Ajya Sah, Amit Sapkota, Smarika Byanju, Shyam Sundar Malla. Prevalence of Iron Deficiency Anemia among Pregnant Women before Iron Supplementation in Kathmandu University Hospital/Dhulikhel Hospital. Journal of Gynecology and Obstetrics. Vol. 2, No. 4, 2014, pp. 54-58. doi: 10.11648/j.jgo.20140204.12

\begin{abstract}
Objective: To determine the prevalence of iron deficiency anemia among pregnant women before iron supplementation in Kathmandu university Hospital/ Dhulikhel Hospital. Study area and population: Fifty three pregnant women who have not started iron supplement and attending OPD of Dhulikhel Hospital were selected for this study. Method and Material: A cluster sampling procedure was applied for this prospective and cross-sectional study. Pregnant women without any ongoing infection were selected. Sociodemographic factors, knowledge, attitude and practices of pregnant women towards iron deficiency anemia were obtained by questionnaires and blood samples were drawn. Hemoglobin, ferritin, Total serum Iron and TIBC were measured. Descriptive statistics was used for data presentation. Results: Among 53 participants overall prevalence of anemia with hemoglobin less than $11 \mathrm{gm} / \mathrm{dl}$ was $37.74 \%$. The prevalence of IDA among pregnant women was $24.52 \%$ and the prevalence of IDA among anemic pregnant women was $65 \%$. The percentage of anemic pregnant women with ferritin $<15 \mathrm{ng} / \mathrm{ml}$ was $70.00 \%$, elevated TIBC $>400 \mu \mathrm{g} / \mathrm{dl}$ was $65 \%$ and total serum iron $<60 \mu \mathrm{g} / \mathrm{dl}$ was $65 \%$. Conclusion: The $65 \%$ prevalence of IDA among anemic pregnant women in the present study suggest that there is high chance of being iron deficient in case of the anemic pregnant women who have not taken iron supplements.
\end{abstract}

Keywords: Anemia, Iron Deficiency, Pregnancy, Dhulikhel Hospital

\section{Introduction}

Micronutrient deficiency may affect growth, cognition and reproductive performance. Of the many micronutrients that are considered lacking in the diets of pregnant women, iron is the most extensively investigated. This is because anemia, attributable to iron deficiency is a major problem in developing countries (WHO, 1992). ${ }^{1}$ Iron deficiency anemia (IDA) is a well documented nutritional deficiency during pregnancy in both developed and developing countries. ${ }^{2}$ IDA is common among women in their reproductive years in particular if the women are poor, pregnant, and members of an ethnic minority. IDA is the hematologic complication of pregnancy and is associated with increased rates of premature birth, low birth weight and prenatal mortality. IDA is known to be an important factor in maternal death, the poor cognitive development of children and decreased work capacity of the mother. ${ }^{3}$

IDA in pregnancy is considered one of the major risk factors contributing to maternal deaths in developing countries; hemorrhage, eclampsia and infections being the three major causes of maternal deaths in Nepal. Risk factors 
including parasite infestation, season, food habits, gestational age, parity, early age at the time of marriage and geographic location for IDA among pregnant women is believed to be more common in the population of Kavrepalanchowk. Dhulikhel Hospital is one of the community based hospital in the district of Kavrepalanchowk and covers a population of 1.9 million. The research on the prevalence of iron deficiency anemia among pregnant women plays an important role to make policy and programmatic decisions to prevent upcoming consequences for both mother and developing fetus.

In order to reduce infant and maternal mortality it is necessary to observe the prevalence of iron deficiency anemia among pregnant women. Thus, the aim of present study, in a random sample of pregnant women from Kathmandu University Hospital/Dhulikhel Hospital, Kavre, Nepal, was to determine the prevalence of IDA among pregnant women before iron supplementation.

\section{Methods}

The study was a prospective, cross sectional study conducted in Kathmandu University Hospital/Dhulikhel Hospital for the duration of 7 weeks. Study subjects included 53 pregnant women attending the Gynecology Department.

Blood samples were collected from all participants by the laboratory technician. The procedure was followed as per operating instruction provided by Department of Clinical Biochemistry, Kathmandu University Hospital/Dhulikhel Hospital.

\subsection{Study Variables}

Dependent variable: Hemoglobin, serum total iron, ferritin, total iron binding capacity.

Independent variable: Sociodemographic factors and socioeconomic factors

Inclusion Criteria:

- Pregnant women to whom iron supplement was not started

- $\quad$ Not suffering from any mental illness, malignant and chronic diseases

\subsection{Exclusion Criteria}

- Pregnant women who suffered from speech and hearing disorders

- Pregnant women who were undergoing treatment for iron deficiency anemia

- $\quad$ Pregnant women who were critically ill

Methodology: Hemoglobin and ferritin were assessed by the procedure followed as per operating instructions provided by Department of Clinical biochemistry, Kathmandu university hospital/Dhulikhel hospital. Cyanmeth hemoglobin method was used for the quantitative determination of Hemoglobin. Enzyme linked Immunosorbent assay (ELISA) was used for the quantitative determination of ferritin.

Total iron and total iron binding capacity was assessed by procedure followed as per operating instruction provided by Department of clinical biochemistry, Kathmandu university hospital/Dhulikhel hospital. The modification of Persijn method was used for the quantitative determination of iron and total iron binding capacity.

\section{Results}

The total sample size of the study was 53 pregnant women.

Table 1 shows the results from the questionnaire used for collecting information from pregnant women. It was observed that the majority of patients were homemakers $(60.38 \%)$ and a majority of them displayed parity (viable gestational age) more than once $(56.60 \%)$ (Table 1$)$.

Table 2 shows the overall statistics of the various parameters observed in pregnant women of the present study.

\subsection{Incidence of Anemia in Pregnant Women of the Present Study}

Table 3 shows the frequency and percentage of the hemoglobin in the present study. The mean hemoglobin was $11.49 \mathrm{gm} / \mathrm{dl}$ with standard deviation of $0.86 .62 .26 \%$ of pregnant women had normal hemoglobin level in the present study. $37.74 \%$ of pregnant women were diagnosed as anemic.

\subsection{Iron Deficiency Assessment in Anemic Pregnant Women in the Present Study}

Ferritin: Table 4 shows the ferritin levels observed in the present study. Among 20 anemic pregnant women, only 30\% of the pregnant women had serum ferritin in normal levels whereas $70 \%$ of the pregnant women had serum ferritin less than $15 \mathrm{ng} / \mathrm{ml}$.

Total Serum Iron levels: Among 20 anemic pregnant women only $35 \%$ of the pregnant women had serum total iron in normal levels whereas $65 \%$ of the women had serum total iron values less than $60 \mu \mathrm{g} / \mathrm{dl}$.

Total iron binding capacity (TIBC): Among 20 anemic pregnant women, only $35 \%$ of the pregnant women had TIBC in normal levels whereas $65 \%$ of the pregnant women had TIBC more than $400 \mu \mathrm{g} / \mathrm{dl}$. Table 5 shows the serum iron and TIBC levels in anemic pregnant women of the present study.

Therefore the prevalence of IDA among pregnant women in 53 samples was $24.53 \%$. The prevalence of IDA among anemic pregnant women in 20 samples was $65 \%$ (Figures $1 \mathrm{a}$ and $\mathrm{b})$. 


\section{Prevalence of IDA among Anemic 20 samples}

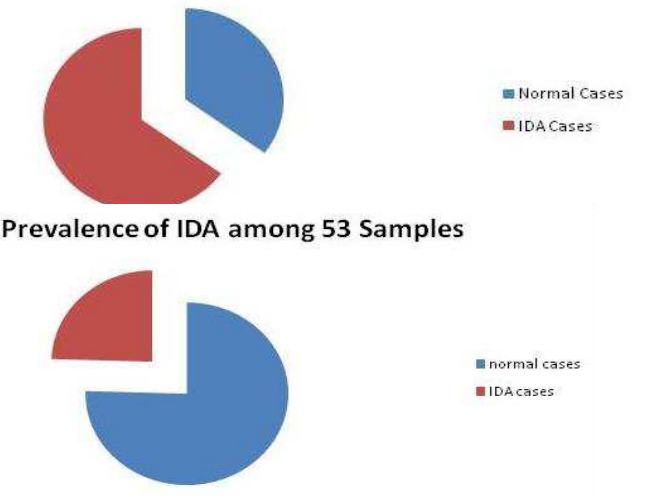

Figure 1a and $\boldsymbol{b}$. Pie chart showing the prevalence of IDA among the women of the present study

Table 1. Results obtained from questionnaire

\begin{tabular}{ll}
\hline Variable & Percentage (\%) \\
\hline Education & \\
Primary education & 16.98 \\
Secondary and above & 83.01 \\
Profession & \\
Housewife & 60.38 \\
Shop & 7.55 \\
Agriculture & 5.66 \\
Teacher & 5.66 \\
Stitch & 3.77 \\
Student & 5.66 \\
Hotel & 5.66 \\
Sub health post & 3.77 \\
Social work & 1.89 \\
Parity & \\
once & 43.396 \\
more than once & 56.60 \\
Duration & \\
1 month & 26.42 \\
2 month & 39.62 \\
3 month & 13.20 \\
4 month & 16.98 \\
5 month & 1.88 \\
7 month & 1.88 \\
\hline
\end{tabular}

Table 2. Statistics for hemoglobin, serum ferritin, total iron, and TIBC levels.

\begin{tabular}{lllll}
\hline Statistics & $\begin{array}{l}\text { Ferritin } \\
\text { Level } \\
\text { (ng/ml) }\end{array}$ & $\begin{array}{l}\text { Total } \\
\text { Iron } \\
\text { level } \\
\text { (mug/dl) }\end{array}$ & $\begin{array}{l}\text { Total Iron } \\
\text { Binding } \\
\text { Capacity } \\
\text { (mug/dl) }\end{array}$ & $\begin{array}{l}\text { Hemoglobin } \\
\text { (gm/dl) }\end{array}$ \\
\hline $\begin{array}{l}\text { Number of } \\
\text { cases }\end{array}$ & 53 & 53 & 53 & 53 \\
Mean & 33 & 85.07 & 345.67 & 11.49 \\
Median & 20 & 83 & 351 & 11.5 \\
Mode & 14 & 59 & 402 & 12 \\
Std. & 28.97 & 23.01 & 57.15 & 0.86 \\
$\begin{array}{l}\text { Deviation } \\
\text { Range }\end{array}$ & 156 & 87 & 285 & 3.9 \\
Minimum & 6 & 55 & 205 & 10 \\
Maximum & 162 & 142 & 490 & 13.9 \\
\multicolumn{5}{l}{ a. Multiple modes exist. The smallest value is shown } \\
\hline
\end{tabular}

Table 3. Hemoglobin levels observed in pregnant anemic women of the present study

\begin{tabular}{lll}
\hline $\begin{array}{c}\text { Hemoglobin } \\
\text { level(gm/dl) }\end{array}$ & Frequency & Percent \\
\hline Normal(11-16) & 33 & $62.26 \%$ \\
more than 16 & 0 & $0.00 \%$ \\
Less than 11 & 20 & $37.74 \%$ \\
Total & 53 & $100.00 \%$ \\
\hline
\end{tabular}

Table 4. Ferritin levels as observed in the present study in anemic pregnant women

\begin{tabular}{lll}
\hline Ferritin(ng/ml) & Frequency & Percent \\
\hline normal(15-180) & 6 & $30.00 \%$ \\
more than 180 & 0 & $0.00 \%$ \\
Less than 15 & 14 & $70.00 \%$ \\
Total & 20 & $100.00 \%$ \\
\hline
\end{tabular}

Table 5. Total Serum Iron and TIBC levels observed in anemic pregnant women of the present study

\begin{tabular}{lll}
\hline Total iron $(\boldsymbol{\mu g} / \mathbf{d l})$ & Frequency & Percent \\
\hline Normal $(60-150)$ & 7 & $35.00 \%$ \\
more than 180 & 0 & $0.00 \%$ \\
Less than 60 & 13 & $65.00 \%$ \\
Total & 20 & $100.00 \%$ \\
TIBC $(\mu \mathrm{g} / \mathrm{dl})$ & & \\
Normal $(250-400)$ & 7 & $35.00 \%$ \\
More than 400 & 13 & $65.00 \%$ \\
Less than 15 & 0 & $0.00 \%$ \\
Total & 20 & $100.00 \%$ \\
\hline
\end{tabular}

Table 6. Prevalence of IDA in pregnant women of the present study

\begin{tabular}{lll}
\hline & Frequency & Percent \\
\hline $\begin{array}{l}\text { Prevalence of IDA among 53 samples } \\
\begin{array}{l}\text { Prevalence of IDA among } 20 \text { anemic } \\
\text { samples }\end{array}\end{array}$ & 13 & $24.52 \%$ \\
\hline
\end{tabular}

\section{Discussion}

Anemia, attributable to iron deficiency is a major problem in developing countries (WHO, 1992). It is estimated that $52 \%$ of pregnant women in developing countries have anemia, whereas this proportion is $23 \%$ in developed countries (WHO, 2001). In case of Nepal, there are very few studies on the prevalence of IDA among pregnant women before iron supplementation in comparison to other studies like anemia among pregnant women. Therefore, it becomes imperative to give immediate attention to pregnant women to address them for iron supplementation.

The difficulty in establishing a precise diagnosis of the iron status of pregnant women represents a complicating 
factor in the understanding of the relationship between maternal and fetal iron levels. The physiological changes that occur during pregnancy (increased plasma volume and erythropoiesis) have a significant impact on hematological and biochemical parameters available for the assessment of iron status. Hemoglobin concentration is the most used parameter to detect anemia in public health care services due to its low cost and available reference standards. Therefore, a combination of several parameters has been proposed in order to improve the diagnosis of iron deficiency (Cook and Finch, 1979)

The $37.74 \%$ prevalence of anemia during pregnancy in the present study was lower than the study conducted by Marahata et al. (2007) (42.6\%) and shah et al. (2001) $(66 \%)$. This may be due the different methods of the estimation of hemoglobin.

In the present study hemoglobin was estimated by Cyanmeth hemoglobin method. This method for estimating hemoglobin is standard and accurate compared to other available methods. The other responsible factors may be environmental condition and different population background such as income, education, food habits and socio demographic factors. In India, another developing country, $88 \%$ of pregnant women were found to be anemic (World Health Organization, UNICEF and UNO, 1998). In the present study, the prevalence of anemia was lower than that of India.

Though the prevalence of IDA in the present study was obviously lower than the prevalence from South-Asian countries (Agarwal et al., 1987; WHO, 1998; Kapil et al., 1999; Bondevik et al., 2000; Dreyfusset et al., 2000) it was higher than found in developing countries. The prevalence of IDA found in other developing countries have been found to be $25-35 \%$ (Karimi et al., 2002).

This may be due to different diagnostic criteria. In the present study IDA was diagnosed by four biochemical parameters such as hemoglobin, serum ferritin, serum total iron, and TIBC.

In the present study with respect to anemic pregnant women, the percentage of ferritin $<15 \mathrm{ng} / \mathrm{ml}$ was present in $70 \%$, elevated $\mathrm{TIBC}>400 \mu \mathrm{g} / \mathrm{dl}$ was present in $65 \%$ and total serum iron $<60 \mu \mathrm{g} / \mathrm{dl}$ was present in $65 \%$ of anemic women.

$\mathrm{S}$-ferritin measurement early in pregnancy is a reliable parameter for judging whether iron supplementation is necessary. Even if S-ferritin is influenced by the plasma dilution, a concentration below 15 microgram $/ 1$ indicates iron deficiency in all stages of pregnancy (Haram et al., 1997).Serum iron measures the amount of iron in the blood. A normal serum iron is $60-170 \mu \mathrm{g} / \mathrm{dl}$. Iron then binds to a protein with the same structure, fetal transferrin and is transported through the bloodstream to fetal tissues. In general, iron-related hematological and biological parameters are markedly higher in umbilical cord than maternal blood (Oski et al., 1984). Lower levels may indicate iron-deficiency anemia or anemia of chronic disease, while higher levels may indicate hemolytic anemia or vitamin B12 deficiency. Total iron binding capacity (TIBC) measures the level of transferrin in the blood. Transferrin is a protein that carries iron in the blood. TIBC calculates how much or how little the transferrin in the body is carrying iron. A higher than normal TIBC is a sign of iron-deficiency anemia.

The $65 \%$ prevalence of IDA among anemic pregnant women in the present study suggest that there is high chance of being iron deficient in case of the anemic pregnant women who have not taken iron supplements.

\section{Conclusion}

On the basis of present study findings, it can be concluded that prevalence of anemia was substantially lower than the national figure for pregnant women. Also the $65 \%$ prevalence of IDA among anemic pregnant women in the present study suggest that there is high chance of being iron deficient in case of the anemic pregnant women who have not taken iron supplements. A larger sample size is needed to confirm the results. But the results of the present study could aid in the development of an iron deficiency anemia program among pregnant women in the district of Kavrepalanchowk that emphasizes on iron supplementation, parasite control and improved diet.

\section{References}

[1] Seshadri Subadra. 2001."Prevalence of micronutrient deficiency particularly of iron, zinc and folic acid in pregnant women in South East Asia. "British Journal of Nutrition 85, Suppl. 2, S87-S92

[2] Aikawa Ritsuko, Khan C Ngyen, Sasaki Satoshi and Binns W Colin. 2005. "Risk factors for iron-deficiency anaemia among pregnant women living in rural Vietnam." Public Health Nutrition: 9(4):443-448

[3] Hadary Jennifer, Cohenand Jere D. Haas. 1999. "Hemoglobin correction factors for estimating the prevalence of iron deficiency anemia in pregnant women residing at high altitudes in Bolivia." Rev Panam Salud Publica/Pan Am J Public Health

[4] Breymann C, Bian XM, Blanco-CapitoLR, Chong C, Mahmud G, Rehman. 2010. "Expert recommendations for the diagnosis and treatment of iron-deficiency anemia during pregnancy and the postpartum period in the AsiaPacific region." Feto maternal hematology unit university hospital

[5] Lusine, Mirzoyan. 1999. "Iron-Deficiency Anemia in Pregnancy: Assessment of Knowledge, Attitudes and Practices of Pregnant Women in Yerevan." Masters diss. American University Of Armenia

[6] Binay Kumar Shah, Lubna A. Baig "Association of anemia with parasitic infestation in pregnant Nepalese women: Result from Hospital based study done in Eastern Nepal." JAMC 17 (1): Jan-Mar 2005

[7] Robbins and Cotran.2010. "Pathologic Basis Of Disease" Haryana, India: Reed Elsevier India Pvt. Ltd. 
[8] Baral KP and Onta, SR. 2009. "Prevalence of anemia amongst adolescents in Nepal: A community based study in rural and urban areas of Morang District." Nepal Med College Journal 11(3); 179-182

[9] Allen H, Lindsay. 2000. "Anemia and iron deficiency: effects on pregnancy outcome."The American Journal Of Clinical Nutrition, 71(5): 1280S-4S

[10] RK Chandyo, TA Strand, RJ Ulvik, RK Adhikari, M Ulak, $\mathrm{H}$ Dixit and H Sommerfelt. 2006. "Prevalence of iron deficiency and anemia among healthy women of reproductive age in Bhaktapur, Nepal." European Journal of Clinical Nutrition, 1-8, Vol: 61, Issue: 2, Pg: 262-269

[11] Dreyfuss ML, Stoltzfus RJ, Shrestha JB, Pradhan EK, LeClerg SC, Khatry SK, Shrestha SR, Katz J, Albonico M, West KP. 2000. "Hookworms, malaria and vitamin A deficiency contribute to anemia and iron deficiency among pregnant women in the plains of Nepal."Department of International Health, The Johns Hopkins School of Hygiene and Public Health,130(10);2527-36

[12] Marahatta, Rita. 2007. Study of anaemia in pregnancy and its outcome in Nepal medical college. Nepal Medical College Journal; 9(4):270-4

[13] Therese O Scholl, Mary L Hediger, Richard L Fisher and Joanne W Shearer .1992. "Anemia vs iron deficiency: increased risk of preterm delivery in a prospective study."The American Journal Of Clinical Nutrition

[14] Fernando E. Viteri: The Consequences of Iron Deficiency and Anaemia in Pregnancy on Maternal Health, the Foetus and the Infant. Department of Nutritional Sciences, University of California at Berkeley

[15] Harper L James, Besa C Emmanuel .2012."Iron deficiency anemia" Medscape reference Drugs disease and procedures.

[16] Meier R Paul, Nickerson H. James, Olson A Kurt. Berg L Richard. Meyer A James.2002." Prevention of iron deficiency anemia in Adolescent and Adult Pregnancies."Clinical Medicine \& Research, 1: 29 - 36
[17] Van Den Broek, Letsky, White and Shenkin. 1998. "Iron status in pregnant women: Which measurements are valid?" British Journal of Haematology 103: 817-824

[18] Lusine, Mirzoyan.1999. "Iron-Deficiency Anemia in Pregnancy: Assessment of Knowledge, Attitudes and Practices of Pregnant Women in Yerevan."American University Of Armenia: 37-49

[19] Hussainab Tabish, Shua Yu Li.2010. "Awareness of iron deficiency anemia among women of Reproductive age in Hubei Province, China." Asian Journal of Medical Sciences 1 (2010):12-13

[20] Rebecca J, Stoltzfus, Michele L, Dreyfuss. "Guidelines for the Use of Iron Supplements to Prevent and Treat iron deficiency anemia." International Nutritional Anemia Consultative Group (INACG)

[21] Mahmoud Abu-Hasira, Amani Waleed. 2007. "Iron Deficiency Anemia among Pregnant Women in Nablus District; Prevalence, Knowledge, Attitude and Practices." Masters diss. An-Najah National University.

[22] Aikawa Ritsuko, Khan C Ngyen, Sasaki Satoshi, Binns W Colin.2005."Risk factors for iron-deficiency anaemia among pregnant women living in rural Vietnam" Public Health Nutrition: 9(4), 443-448

[23] WHO-World Health Organization. Iron deficiency anaemia -assessment, prevention and control: a guide for programme managers. Geneva; 2001

[24] Haram K, Hervig T, Ulvik RJ. "Hemoglobin, iron deficiency and anemia in pregnant women. Diagnostic aspects." Tidsskr Nor Laegeforen. 1997 Mar 10;117 (7):962-6.

[25] Oski FA, Naiman JL, Stockman JA. Hematologia do recémnascido. São Paulo: Manole; 1984.

[26] Karimi M, Kadivar R, Yarmohammadi H.Assessment of the prevalence of iron deficiency anemia, by serum ferritin, in pregnant women of Southern Iran. Med Sci Monit. 2002 Jul; 8(7):CR488-92. 